Rev. Int. Contam. Ambie. 37, 119-131, 2021

https://doi.org/10.20937/RICA.53718

\title{
CAMBIOS EN LOS BIOMARCADORES DE LA FRACCIÓN DE HIDROCARBUROS SATURADOS EN UN ENSAYO DE BIORREMEDIACIÓN CON UN CRUDO EXTRAPESADO
}

Changes in biomarkers from a saturated hydrocarbon fraction in a bioremediation test with extra heavy oil

\author{
Liliana LÓPEZ* y Carmen INFANTE
}

\author{
Laboratorio de Geoquímica Orgánica, Instituto de Ciencias de la Tierra (ICT), Facultad de Ciencias, Universidad \\ Central de Venezuela, Caracas, Venezuela 1053. \\ *Autora para correspondencia; email: liliana.lopez@ciens.ucv.ve
}

(Recibido: agosto de 2019; aceptado: agosto de 2020)

Palabras clave: biorremediación, crudo extrapesado, biomarcadores, biodegradación, fosas petroleras.

\section{RESUMEN}

Se evaluaron los biomarcadores en la fracción de hidrocarburos saturados ( $n$-alcanos, pristano, fitano, terpanos y esteranos) en un ensayo de biorremediación de 90 días con un crudo extrapesado ( $\left.10^{\circ} \mathrm{API}\right)$. Con base en la presencia de una muestra compleja no resuelta (MCNR), deficiencia de $n$-alcanos, pristano, fitano y presencia de hopanos desmetilados (25-norhopanos), se determinó que el crudo original está biodegradado. La detección de $n$-alcanos, pristano y fitano (más sensibles a la biodegradación), junto con 25-norhopanos (menos sensibles a biodegradación) sugiere que el crudo analizado es el resultado de mezclas de distintas cargas de crudo en el yacimiento. Después de 90 días del ensayo de biorremediación se encontró el siguiente orden de biodegradación en el crudo para los hopanos extendidos $(\mathrm{R}+\mathrm{S})$ : $\mathrm{C}_{35}>\mathrm{C}_{34}<\mathrm{C}_{33}>\mathrm{C}_{32}>\mathrm{C}_{31}$. El cambio en la distribución de los hopanos extendidos respecto al crudo original indica una mayor alteración para $\mathrm{C}_{32}$ y $\mathrm{C}_{35}$, y en los isómeros $22 \mathrm{~S}$ y $22 \mathrm{R}$ una degradación selectiva para $\mathrm{C}_{33} 22 \mathrm{~S}$. Se sugiere alteración de los terpanos tricíclicos debido a la variación en la intensidad de la señal en el crudo original y la determinada a los 90 días. En los esteranos no se observó alteración al final del ensayo; sólo variación en la intensidad de las señales que sugiere disminución en su concentración. Durante el ensayo de biorremediación se produjo la remoción de los alcanos $<n-C_{25}$, sin alteración de los $>n$ - $\mathrm{C}_{25}$ y los isoprenoides pristano y fitano. Adicionalmente, los terpanos tricíclicos, hopanos extendidos y esteranos fueron alterados.

Key words: bioremediation, extra heavy oil, biomarkers, biodegradation, pit wastes.

\begin{abstract}
Biomarkers in the saturated hydrocarbon fraction ( $n$-alkanes, pristane, phytane, terpanes, and steranes) were evaluated in a 90-day bioremediation assay with extra heavy crude $\left(10^{\circ} \mathrm{API}\right)$. Based on the presence of an unresolved complex mixture (UCM), deficiency of $n$-alkanes, pristane, phytane and presence of demethylated hopanes (25-norhopanes), it was determined that the original oil is biodegraded. Detecting $n$-alkanes, pristane and phytane (more susceptible to biodegradation), along with 25-norhopanes (less susceptible to biodegradation), suggests that the crude oil analyzed is the result of mixtures of
\end{abstract}


several episodes of charging and biodegradation in the reservoirs. In crude oil after 90 days of the bioremediation test, the following order of biodegradation was found for the extended hopanes $(\mathrm{R}+\mathrm{S}): \mathrm{C}_{35}>\mathrm{C}_{34}<\mathrm{C}_{33}>\mathrm{C}_{32}>\mathrm{C}_{31}$. The change in the distribution of the extended hopanes related to the original oil indicates a greater alteration of $\mathrm{C}_{32}$ and $\mathrm{C}_{35}$, and in the $22 \mathrm{~S}$ and $22 \mathrm{R}$ isomers, a selective degradation for $\mathrm{C}_{33} 22 \mathrm{~S}$. Alteration of tricyclic terpanes is suggested, due to the variation in signal intensity in the original oil and that determined at 90 days. At the end of the test no alteration in steranes was observed, with only variation in signal intensity that suggests decreased concentration of these compounds. During the bioremediation test, $<n-\mathrm{C}_{25}$ alkanes were removed without altering $>n-\mathrm{C}_{25}$, and pristane and phytane isoprenoids. Additionally, tricyclic terpanes, extended hopanes and steranes were altered.

\section{INTRODUCCIÓN}

La biorremediación es una tecnología ampliamente usada para la limpieza de suelos contaminados por hidrocarburos. Se basa en el proceso de biodegradación, equivalente a la transformación y mineralización completa o parcial de los hidrocarburos mediante acción microbiana. Esto permite disminuir la concentración de hidrocarburos en el suelo para llegar a determinados criterios de limpieza (Infante 2001, 2005, Erdogan y Karaca 2011, Ali et al. 2020). El proceso es más efectivo en componentes de baja masa molecular y crudos de alta gravedad API, el cual ha sido estudiado en diferentes condiciones de $\mathrm{C} / \mathrm{N}, \mathrm{C} / \mathrm{P}, \mathrm{C} / \mathrm{K}$, agentes estructurantes, textura del suelo, bioaumentación y bioestimulación, entre otros factores (Infante et al. 2010a, b, Ali et al. 2020). Sin embargo, se conoce poco sobre la transformación completa o parcial de los hidrocarburos en crudos extrapesados sometidos a ensayos de biorremediación, específicamente para los biomarcadores (pristano, fitano, $n$-alcanos, terpanos y esteranos) que se encuentran en la fracción de los hidrocarburos saturados.

La mayoría de estos crudos $\left(<10^{\circ}\right.$ API $)$ han sufrido biodegradación en el yacimiento, debido a que los compuestos de menor masa molecular y menor condensación han sido degradados por bacterias (Volkman et al. 1983a, Peters y Moldowan 1993). Por ello, no es sorprendente que en suelos contaminados con crudos pesados y extrapesados existan compuestos más recalcitrantes al proceso de biorremediación que en suelos contaminados con crudos livianos y medianos y, en consecuencia, no se encuentre una disminución significativa de los hidrocarburos totales en el tiempo (Infante et al. 2010a, b). Este mismo comportamiento se aplica también a los depósitos de desechos petroleros o pasivos ambientales en las denominadas fosas, agujeros o piscinas. En el pasado, estos depósitos se usaron como práctica común en la mayoría de los países con explotación petrolera para almacenar diferentes tipos de desechos, como suelos de derrames de crudo de diferentes API, borras o fondos de tanques y cortes de perforación (Infante 2001, 2005). Con el transcurso de los años estos desechos experimentan cambios físicos y químicos por procesos de evaporación, fotoxidación y biodegradación (Ali et al. 2020).

En Venezuela, una alta proporción de la explotación petrolera está ubicada en el oriente del país, y en los campos petroleros de esa región e encuentra la mayor proporción de crudos pesados $\left(22\right.$ a $10.1^{\circ}$ API) y extrapesados $\left(<10^{\circ} \mathrm{API}\right)$, muchos de ellos producto del proceso de biodegradación que ocurre en los yacimientos (Alberdi et al. 1996, López y Lo Mónaco 2010, López 2014, López et al. 2015). Por lo tanto, en esa zona los desechos que se encuentran en fosas petroleras o los que pueden ser el resultado de derrames corresponden a crudos cuya composición química presenta alteración por biodegradación.

La biodegradación en el yacimiento produce crudos caracterizados por disminución de su gravedad API, incremento en la viscosidad, concentración de resinas, asfaltenos, azufre, metales como vanadio y níquel, disminución de las concentraciones de hidrocarburos saturados y aromáticos, y cambios en la composición molecular relacionados con alteración de $n$-alcanos, isoprenoides acíclicos (pristano y fitano), terpanos y esteranos (Wenger et al. 2002, Peters et al. 2005, Larter et al. 2006). El grado de alteración de los hidrocarburos en el crudo que se encuentra en un yacimiento de petróleo se mide en función de su sensibilidad a la biodegradación y de las escalas de biodegradación propuestas (Volkman 1983a, b, 1984, Peters y Moldowan 1993).

Debido a lo anterior, estudiar el comportamiento de los biomarcadores en un ensayo de biorremediación de un suelo impactado por un crudo extrapesado, lleva a comprender mejor las transformaciones químicas que pueden ocurrir durante el proceso de biodegradación. Esto es de interés en zonas impactadas 
con crudos pesados o extrapesados o con desechos petrolizados de fosas antiguas, cuyas fracciones biodegradables son pocas. Por lo tanto, su comportamiento es diferente al observado en crudos livianos (30 a $22.1^{\circ} \mathrm{API}$ ) y medianos ( $\geq 30.1$ ), en los cuales la biorremediación suele ser más efectiva (García et al. 2012, Córdova et al. 2013).

En este trabajo se estudian los cambios en los biomarcadores de la fracción de hidrocarburos saturados ( $n$-alcanos, pristano y fitano, terpanos y esteranos) en un ensayo de biorremediación en microcosmos de suelo, de un crudo extrapesado $\left(10^{\circ} \mathrm{API}\right)$ de la Faja Petrolífera del Orinoco, específicamente del área de Ayacucho, Venezuela. La información obtenida de esta investigación aporta datos interesantes para conocer la evolución por biorremediación de crudos biodegradados. Sus cambios pueden determinarse a través de los biomarcadores por transformaciones internas a nivel molecular del crudo. Esto es de utilidad al aplicar biorremediación a un crudo extrapesado o en desechos de fosas petrolizadas, donde son muy poco conocidos los cambios en crudos de baja gravedad API.

\section{MUESTRAS Y MÉTODOS}

\section{Muestras}

El suelo empleado para los ensayos de biorremediación es de textura franco arenoso y fue recolectado en la Facultad de Ciencias de la Universidad Central de Venezuela, específicamente en el área de la Escuela de Física. Se recolectaron 10 muestras de los primeros $20 \mathrm{~cm}$ de suelo en varios puntos al azar, para obtener una muestra compuesta de $1.5 \mathrm{~kg}$. La muestra de crudo utilizada para los ensayos de biorremediación corresponde a crudo extrapesado de $10^{\circ}$ API del área de Ayacucho (Hamaca), en la Faja Petrolífera del Orinoco.

\section{Tratamiento de las muestras y análisis Caracterización del suelo}

El suelo recolectado fue disgregado y se eliminaron los fragmentos de rocas para obtener una muestra lo más homogénea posible. Las hojas u otro material orgánico presentes en el suelo se fragmentaron lo más finamente posible y se dejaron dentro de la muestra de suelo como agentes estructurante, para que de esta manera los restos vegetales presentes facilitaran la biorremediación al mejorar la estructuración del suelo. La composición mineralógica de la muestra se evaluó por el método de polvo cristalino en un difractómetro D-8 Advance AXS (Bruker, Alemania).
Para determinar las fases minerales presentes en cada sólido se utilizó el programa del Centro Internacional de Datos de Difracción (Wong-Ng et al. 2001). Las concentraciones de carbono total (CT) se determinaron en un equipo LECO (C-144) y para el carbono carbonático (CI) se utilizó un calcímetro de Bernard (Hesse 1971). El porcentaje de humedad se cuantifico por diferencia de peso entre el peso obtenido de alícuotas del suelo original y su peso después de secarse en estufa por $24 \mathrm{~h}$ a $110^{\circ} \mathrm{C}$ (tres replicas).

\section{Caracterización del crudo}

Antes de someter al crudo a la técnica de análisis se realizó un tratamiento previo para la eliminación de agua y sedimentos. El procedimiento se realizó en dos pasos, consistentes en agregar tolueno a las muestras de crudo en una relación 1:1 y centrifugarlas a $1500 \mathrm{rpm}$ durante $30 \mathrm{~min}$. Posteriormente la solución de crudo con tolueno se evaporo al vacío a una temperatura de $80^{\circ} \mathrm{C}$ para la eliminación del solvente y obtención del crudo limpio. Este tratamiento elimina los hidrocarburos de baja masa molecular, como los compuestos volátiles. Sin embargo, los crudos pesados y extrapesados contienen bajos niveles de compuestos volátiles, y los pocos que están presentes se pierden al momento de la toma de muestras de crudo a cabezal de pozo o durante el almacenamiento (Brown et al. 2017).

\section{Ensayos de biorremediación}

Se siguió un protocolo de biorremediación similar al utilizado en otras investigaciones con diferentes tipos de crudos (Infante 2005), texturas de suelos (García et al. 2012), dosis y tipos de agentes estructurantes, así como diferentes relaciones $\mathrm{C} / \mathrm{N}, \mathrm{C} / \mathrm{P}$ y $\mathrm{C} / \mathrm{K}$, bioaumentación y bioestimulación (Córdova et al. 2013, Infante et al. 2010a, b), sólo que a una escala de microcosmos menor, empleando las mejores condiciones abióticas y bióticas (bioestimulación) para la biorremediación. Para ello se pesaron tres muestras de suelo de $150 \mathrm{~g}$ cada una, las cuales se colocaron en vasos de precipitado de $1000 \mathrm{~mL}$ que conformaron los microcosmos. Una vez colocados los $150 \mathrm{~g}$ de suelo se agregaron 1.5 $\mathrm{g}$ de urea equivalentes a 0.69 gramos de nitrógeno, la cual fue incorporada al suelo, con posterior adición de $10 \mathrm{~mL}$ de agua y mezcla. Enseguida se procedió a añadir $10 \mathrm{~g}$ del crudo Ayacucho a cada uno de los tres microcosmos. Para obtener una mejor mezcla del crudo-suelo, se fueron agregando pequeñas cantidades de crudo seguido de mezcla, hasta obtener una distribución lo más homogénea posible. Debido a la baja gravedad API del crudo Ayacucho, 
la cantidad añadida fue transvasada utilizando una varilla de vidrio. El porcentaje de humedad de los ensayos se mantuvo entre 30 y $60 \%$ de la capacidad de campo, agregando $10 \mathrm{~mL}$ de agua cada tercer día hasta la finalización del ensayo. De igual manera fueron aireados diariamente, empleando una varilla de vidrio, con la cual se mezcló cada microcosmos a fin de obtener oxigeno del intercambio con el ambiente.

\section{Toma de muestras de los microcosmos}

Se tomaron alícuotas de $10 \mathrm{~g}$ de cada uno de los tres microcosmos, a los tiempos denominados $\mathrm{t}_{1}, \mathrm{t}_{30}$, $\mathrm{t}_{60}$ y t90, correspondientes a uno, 30,60 y 90 días de los ensayos, respectivamente. Al crudo original le corresponde $\mathrm{t}_{0}$ ( (sin suelo).

\section{Separación de crudo durante el ensayo de biorre- mediación}

Las alícuotas de $10 \mathrm{~g}$ de suelos tomadas en los ensayos a los distintos tiempos de recolección se colocaron en un embudo Buchner con membrana millipore $(0.5 \mathrm{~mm})$, acoplado a matraz del vidrio de succión del vacío, El crudo se extrajo del suelo mediante adición de $150 \mathrm{~mL}$ de cloruro de metileno $\left(\mathrm{CH}_{2} \mathrm{Cl}_{2}\right)$. Posteriormente, la mezcla solvente-crudo se colocó en un balón de fondo plano de boca esmerilada de $250 \mathrm{~mL}$ y el cloruro de metileno se eliminó en un equipo de evaporación al vacío (rotavapor) a $40{ }^{\circ} \mathrm{C}$.

\section{Determinación de carbono orgánico total rema- nente}

Una vez realizada la extracción del crudo en los suelos a los tiempos $\mathrm{t}_{1}, \mathrm{t}_{30}, \mathrm{t}_{60}$ y $\mathrm{t}_{90}$ se determinó la concentración de carbono orgánico en el suelo, lo cual equivale al carbono orgánico total (COT) remanente. Esta medida se realizó en un equipo marca Leco modelo C-144. Para ello se pesaron aproximadamente $170 \mathrm{mg}$ de suelo tomado en los diferentes tiempos con $100 \mathrm{mg}$ de acelerador (Com Cat) en una celda de combustión de cuarzo-mullita. Luego de pesar los patrones estándar Leco y las muestras, se analizaron primero los patrones para obtener la curva de calibración y posteriormente las muestras. La lectura obtenida de la combustión de carbono se detectó a través de una celda de infrarrojo que, al relacionar con el peso de la muestra, permitió calcular la concentración de CT o COT en porcentaje $\mathrm{m} / \mathrm{m}$.

Separación de la fracción de hidrocarburos saturados del crudo

Se realizó la separación en maltenos y asfaltenos del crudo original $\left(\mathrm{t}_{0}\right)$ y el extraído a los 90 días $\left(\mathrm{t}_{90}\right)$ del ensayo de biorremediación, utilizando para ello $n$-heptano en frío en una relación crudo/n-heptano de $1 / 40(\mathrm{~m} / \mathrm{v})$, con posterior centrifugación $(1500 \mathrm{rpm}$, $30 \mathrm{~min}$ ) y lavado, correspondiendo el sobrenadante a la fracción de maltenos. Posteriormente, de la fracción de maltenos fueron separados los hidrocarburos saturados por cromatografía de adsorción en columnas de $20 \mathrm{~cm}$ de largo y $1.5 \mathrm{~cm}$ de diámetro, empacadas con $20 \mathrm{~g}$ de alúmina previamente activada durante $8 \mathrm{~h}$ a $280{ }^{\circ} \mathrm{C}$ y $25 \mathrm{~mm}$ de $\mathrm{Hg}$. Para la elución de los hidrocarburos saturados se utilizaron $35 \mathrm{~mL}$ de $n$-hexano. Los hidrocarburos saturados obtenidos fueron nuevamente eluidos con $35 \mathrm{~mL}$ de $n$-hexano a través de columnas empacadas como las descritas anteriormente, con el objeto de evitar posible contaminación con la fracción de los hidrocarburos aromáticos del crudo durante la separación y para su posterior análisis por cromatografía de gases acoplado a masas.

\section{Análisis de los biomarcadores de la fracción de hidrocarburos saturados}

Los hidrocarburos saturados fueron analizados por cromatografía de gases acoplada a espectrometría de masas en un cromatógrafo de gases (CG-EM) de Agilent Technologies, modelo 6890N Network DCSystem, acoplado a un espectrómetro de masas MS Agilent 5973 Network Mass Selective Detector, equipado con una columna capilar DB1 $(60 \mathrm{~m} \times 0.25$ $\mathrm{mm} \times 0.25 \mu \mathrm{m}$ ). Las condiciones de análisis por CGEM para los hidrocarburos saturados fueron: temperatura del inyector de $275^{\circ} \mathrm{C}$ y temperatura inicial de horno de $80^{\circ} \mathrm{C}$ durante 4 min con una rampa de calentamiento de $2{ }^{\circ} \mathrm{C} / \mathrm{min}$ hasta una temperatura final de $280^{\circ} \mathrm{C}$ por $10 \mathrm{~min}$. Se utilizó He como gas de arrastre a un flujo de $1 \mathrm{~mL} / \mathrm{min}$. Para la obtención de los cromatogramas de masas o fragmentogramas se utilizó el método de los iones monitoreados, analizando los iones $m / z=113$ ( $n$-alcanos e isoprenoides acíclicos pristano y fitano), 191 (terpanos), 177 (25-norhopanos), 217 y 218 (esteranos). La identificación de los biomarcadores se llevó a cabo por comparación con la muestra de referencia de nuestro laboratorio (crudo SSW-43 de la subcuenca de Barinas). Las relaciones de los biomarcadores utilizados para la evaluación del nivel de biodegradación se calcularon utilizando las áreas determinadas en los cromatogramas de masa correspondientes.

\section{RESULTADOS Y DISCUSIÓN}

\section{Caracterización del suelo}


La composición mineralógica, del suelo corresponde a cuarzo, caolinita y moscovita. El porcentaje de humedad fue de $21 \pm 1 \%$. La concentración de CT obtenida fue de $5.1 \pm 0.2 \% \mathrm{~m} / \mathrm{m}$ (resultado de tres medidas). Se asume que la concentración de CT determinada en el equipo Leco corresponde al COT, ya que al determinar la composición mineralógica no se detectó calcita ni otro tipo de carbonato de calcio; por otra parte, la concentración de carbono CI medida con el calcímetro de Bernard (Hesse 1971) está por debajo del límite de detección de esta técnica $(0.5 \%)$.

\section{Evaluación del COT remanente después la re- moción del crudo durante el ensayo de biorre- mediación}

Las concentraciones de COT en el suelo se determinaron en alícuotas de muestra captadas en los ensayos a $1,30,60$ y 90 días $\left(t_{1}, t_{30}, t_{60}\right.$ y $t_{90}$, respectivamente). En estos resultados se puede observar un leve incremento en la concentración de COT del suelo en $\mathrm{t}_{1}(5.7 \%)$, respecto a su valor original de $5.1 \%$ (suelo sin crudo) (Fig. 1). Esto se debe posiblemente a la variación natural de las muestras o al crudo que queda en suelo que no se puede extraer completamente con el cloruro de metileno, además de la posible extracción de lípidos y otros compuestos orgánicos propios de la materia orgánica del suelo (restos vegetales, carbono microbiano). A partir de

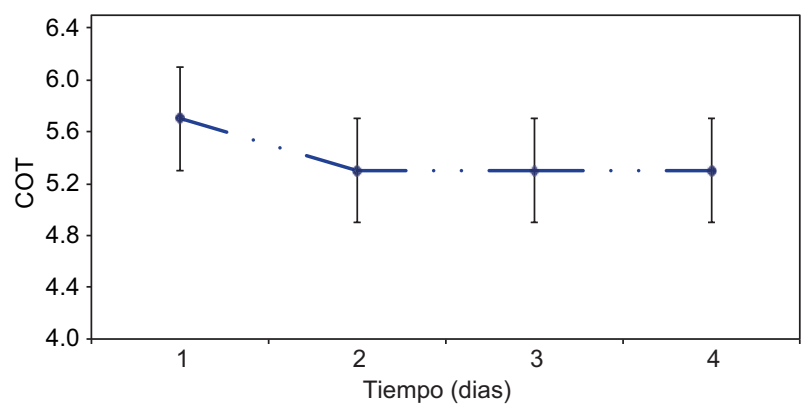

Fig. 1. Variación de las concentraciones de carbono orgánico total (COT) en el suelo remanente (después de extracción de crudo) a los 1, 30, 60 y 90 días.

los 30 días y hasta los 90 , permanece constante, indicando que no hay cambios en el CT remanente.

\section{Composición del crudo Ayacucho}

El crudo Ayacucho es extrapesado ( $\left.10^{\circ} \mathrm{API}\right)$, presenta baja concentración de hidrocarburos saturados (6.4\%) y aromáticos $(45.8 \%)$, y está enriquecido con compuestos NSO (resinas + asfaltenos [47.7\%]). De acuerdo con su composición de hidrocarburos saturados y aromáticos, resinas y asfaltenos (SARA) se clasifica como un crudo aromático-asfáltico (López y Lo Mónaco 2017). A partir del análisis de la distribución de $n$-alcanos $(m / z=113)$ se determinó que el crudo esta biodegradado con base en: 1) presencia de una mezcla compleja no resuelta (MCNR); 2) deficiencia de $n$-alcanos e isoprenoides acíclicos (pristano y fitano), y 3) presencia de hopanos desmetilados o 25-norhopanos. Por otra parte, para los esteranos y terpanos no hay evidencias de biodegradación, con base en: 1) presencia de los esteranos regulares de $\mathrm{C}_{27}, \mathrm{C}_{28}$ y $\mathrm{C}_{29}$; 2) presencia de los terpanos tricíclicos, terpanos pentacíclicos y hopanos extendidos. A continuación se ofrece la explicación de cada uno

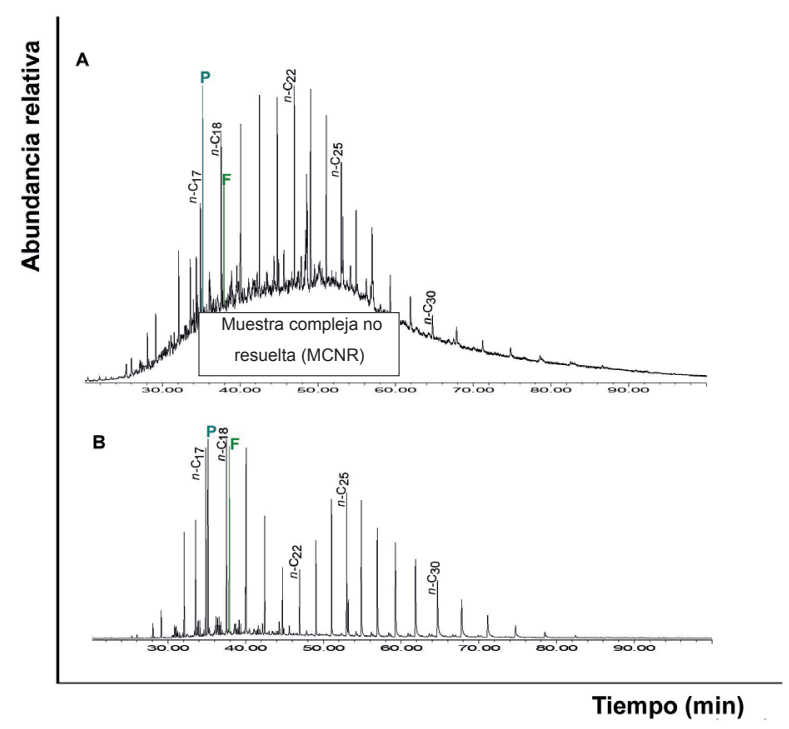

Fig. 2. Distribución de $n$-alcanos e isoprenoides acíclicos pristano (P) y fitano (F) $(\mathrm{m} / \mathrm{z}=113)$. (a) Crudo Ayacucho original, (b) crudo Ayacucho luego de 90 días de biorremediación.

de estas evidencias, las cuales permiten determinar el nivel de biodegradación del crudo original que se presenta en las figuras $2 \mathbf{a}$ y $\mathbf{5 a}$.

La presencia de MCNR o joroba en el cromatograma, es una típica evidencia de biodegradación (Fig. 2a). Esta MCNR consiste en compuestos orgánicos que no pueden ser identificados en las condiciones de análisis utilizadas para la separación de los $n$-alcanos a través del $m / z=113$, y consta de compuestos biorresistentes como saturados cíclicos, aromáticos, naftenoaromáticos, y compuestos polares que no pueden separarse mediante la técnica de cromatografía (Tissot y Welte 1984, Killops y al-Juboori 


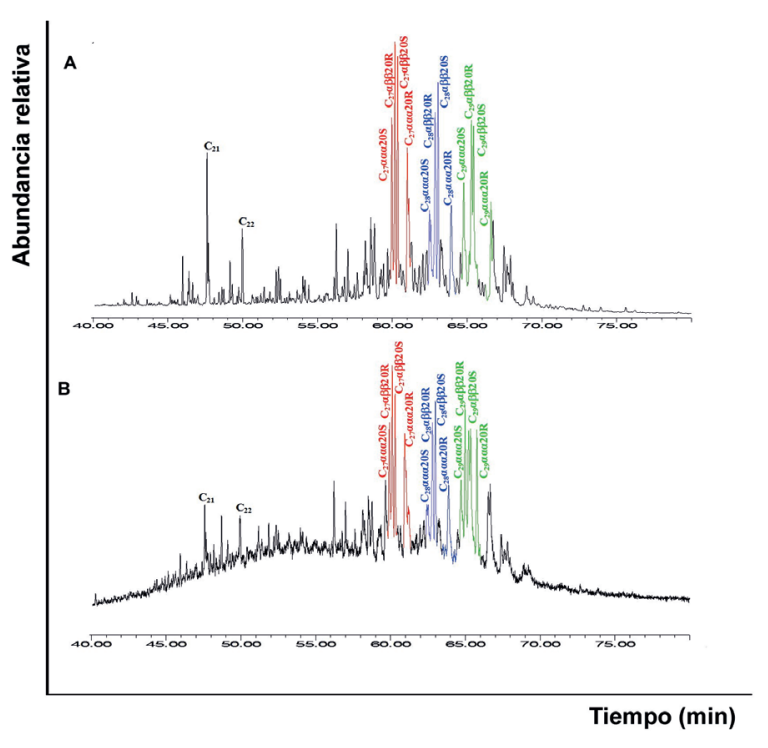

Fig. 3. Distribución de esteranos $(m / z=217)$. (a) Crudo Ayacucho original, (b) crudo Ayacucho luego de 90 días de biorremediación.

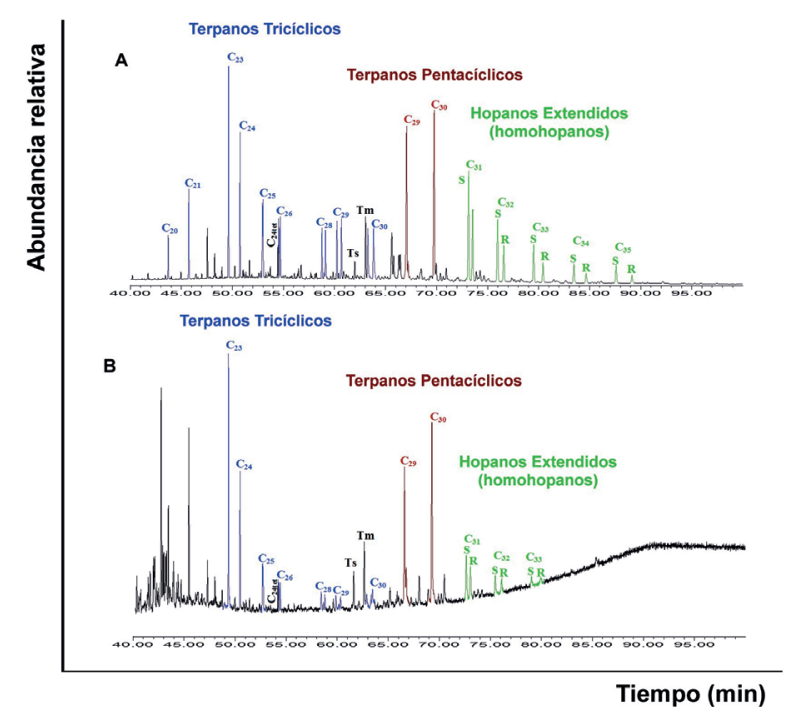

Fig. 4. Distribución de terpanos $(m / z=191)$. (a) Crudo Ayacucho original, (b) crudo Ayacucho luego de 90 días de biorremediación. $\mathrm{C}_{24 t e t}$ : terpano tetracíclico; Ts: $\mathrm{C}_{27} \alpha-22,29,30-$ trisnorneohopano; Tm: $\mathrm{C}_{27} \alpha-22,29,30$-trisnorhopano.

1990, Gough y Rowland 1990, Sutton et al. 2005).

Sin embargo, sobre la MCNR se observan, en señales de baja intensidad, $n$-alcanos entre $n$ - $\mathrm{C}_{17}$ y $n-\mathrm{C}_{34}$, y los isoprenoides acíclicos pristano $(\mathrm{P})$ y fitano (F) (Fig. 2a), lo que sugiere que no todos los $n$-alcanos (al igual que los isoprenoides $\mathrm{P}$ y $\mathrm{F}$ ) han sido biodegradados. Para este crudo, basado en el pa- trón de distribución $\mathrm{m} / \mathrm{z}=217$ de todos los isómeros ( $\alpha \alpha \alpha 20 \mathrm{~S}, \alpha \alpha \alpha 20 \mathrm{R}, \alpha \mathrm{bb} 20 \mathrm{~S}$ y $\alpha \mathrm{bb} 20 \mathrm{R})$, para cada uno de los esteranos regulares en el intervalo de $\mathrm{C}_{27}$ a $\mathrm{C}_{29}$ (Fig. 3a), se determinó que no fueron biodegradados. De igual manera, el patrón de distribución para los terpanos $(m / z=191)$, donde se detectaron los terpanos triciclos en el intervalo de $\mathrm{C}_{20}$ a $\mathrm{C}_{30}$, los pentacíclicos (hopanos) $\mathrm{C}_{29}$ y $\mathrm{C}_{30}$ (hopanos) y los hopanos extendidos (homohopanos) de $\mathrm{C}_{31}$ a $\mathrm{C}_{35}$, indica que no ocurrió biodegradación para esta fracción (Fig. 4a) Sin embargo, la presencia de 25-norhopanos, en particular en el $m / z=177$, donde se identifica la serie de 25-horhopanos que incluye los $\mathrm{C}_{27}$-trisnorhopano (TNH), $\mathrm{C}_{28}$-dinorhopano (DNH), $\mathrm{C}_{29}$-norhopano (NH) y la serie completa $\mathrm{C}_{30}-\mathrm{C}_{34}$ norhomohopanos (Fig. 5a), sugiere alteración por biodegradación para los hopanos y hopanos extendidos.

La alteración de los hopanos regulares por remoción bacteriana del grupo metilo en posición C-10, produce los 25-norhopanos (10-desmetilhopanos u hopanos desmetilados), una serie de compuestos que están presentes en muchos crudos biodegradados. Esta alteración ocurre para los hopanos $\mathrm{C}_{28}, \mathrm{C}_{29} \mathrm{y}$ $\mathrm{C}_{30}$, y los homohopanos de $\mathrm{C}_{31}$ a $\mathrm{C}_{35}$ (Peters et al. 2005). Para los homohopanos $C_{31}$ a $C_{35}$, el orden de alteración por biodegradación corresponde a: $\mathrm{C}_{35}$ $>\mathrm{C}_{34}>\mathrm{C}_{33}>\mathrm{C}_{32}>\mathrm{C}_{31}$. La identificación de estos compuestos se determina mediante la disminución de la señal en el fragmentograma de $m / z=191$, la cual es paralela al incremento de la señal de los 25 -norhopanos en el cromatograma de masas de $m / z=177$. Con la pérdida de un grupo $\mathrm{CH}_{3}$ y la adición de un átomo de $\mathrm{H}$, el fragmento de $m / z=191$ corresponde ahora a un fragmento de $m / z=177$, observándose por lo tanto una disminución del tiempo de retención por la disminución de la masa molecular del compuesto (López 2013).

De los resultados obtenidos a partir de la distribución de los biomarcadores ( $n$-alcanos, pristano, fitano, esteranos y terpanos) y utilizando la escala de biodegradación de Peters y Moldowan (1993), se puede interpretar la historia de llenado del yacimiento y el proceso de biodegradación del crudo. Como en este crudo se observan $n$-alcanos, así como los isoprenoides acíclicos pristano y fitano (más sensibles a la biodegradación) junto con 25-norhopanos producto de alteración de los hopanos (formados a un nivel de biodegradación más avanzado [Peters y Moldowan 1993]), se sugiere que la distribución observada para los biomarcadores es el resultado de la mezcla de una primera carga de crudo en el yacimiento cuya biodegradación alcanzó un nivel de 7 , apoyado en la presencia de 25-norhopanos, de acuerdo con la 




Fig. 5. Distribución de terpanos $(m / z=191)$ y hopanos desmetilados o 25 -norhopanos $(\mathrm{m} / \mathrm{z}=177)$. (a) Crudo Ayacucho original, (b) crudo Ayacucho luego de 90 días de biorremediación. D27TNH: C27-trisnorhopano; D28DNH: C28-dinorhopano; D29NH: C29-norhopano (NH).

escala de biodegradación de Peters y Moldowan (1993). Posteriormente ocurrió una segunda carga de crudo en el yacimiento (el cual probablemente fue sometido sólo a una biodegradación incipiente), donde aún se observan $n$-alcanos y los isoprenoides acíclicos pristano y fitano (López et al. 2015). Estas características también pueden ser consecuencia de la difusión y mezcla de crudos, los cuales se encuentran en yacimientos con acumulaciones de poco espesor que han sido biodegradados en diferentes grados. Este caso puede aplicarse al crudo de Ayacucho, ya que en muchas zonas de la Faja Petrolífera del Orinoco, las arenas impregnadas de crudo son de poco espesor y tienen contacto continuo con aguas (Casas et al. 2007, Pardo et al. 2007).

El estudio de los biomarcadores ( $n$-alcanos, pristano, fitano, esteranos y terpanos) del crudo Ayacucho, refleja su complejidad por el proceso de biodegradación, lo que es común en muchas cuencas petrolíferas a nivel mundial y hace más realista el uso de este tipo de crudo en ensayos de biorremediación para los desechos que se encuentran en fosas petroleras, las cuales generalmente son mezclas de crudos de diferentes orígenes y/o con distintos estado de alteración por biodegradación (Moldowan et al. 1995, Larter et al. 2003, 2006).

\section{Caracterización del crudo Ayacucho extraído luego del ensayo de biorremediación}

La distribución de $n$-alcanos y de los isoprenoides acíclicos fitano (F) y pristano (P), luego de los 90 días de biorremediación $\left(\mathrm{t}_{90}\right)$ presenta pérdida de MCNR, tal como se puede observar en la figura $\mathbf{2 b}$, donde la línea base del cromatograma de masas $\mathrm{m} / \mathrm{z}=113$ es completamente normal, sin levantamiento. Tal como se mencionó anteriormente, la MCNR en un cromatograma representa una mezcla de compuestos no resueltos en las condiciones utilizadas en cromatografía de gases para el análisis de la fracción de hidrocarburos saturados de crudos, que se consideran el producto de la biodegradación del crudo en el yacimiento (Tissot y Welte 1984, Killops y al-Juboori 1990, Sutton et al. 2005). La ausencia de MCNR, una vez que el crudo es sometido a los 90 días de biodegradación para la biorremediación del suelo, no puede explicarse a partir de los resultados obtenidos 
en este estudio, ya que de acuerdo con la literatura (Killops y al-Juboori 1990, Sutton et al. 2005) la complejidad y escaso conocimiento de los compuestos incluidos en la fracción representada por la MCNR, dificultan una explicación sobre su ausencia luego de los 90 días de biorremediación. Sin embargo, en $m / z=113$ se evidencia una disminución en la intensidad de la señal correspondiente a los $n$-alcanos $<$ $n-\mathrm{C}_{25}$, lo cual puede constatarse de acuerdo con la relación $\mathrm{C}_{17}-\mathrm{C}_{25} / \mathrm{C}_{26}-\mathrm{C}_{34}$, que disminuye luego de los ensayos de biorremediación (Cuadro I). De igual manera, se observa disminución en la señal de los $n$-alcanos $n$ - $\mathrm{C}_{17}$ y $n$ - $\mathrm{C}_{18}$ respecto a pristano y fitano, con incremento en las relaciones $\mathrm{P} / n-\mathrm{C}_{17}$ y $\mathrm{F} / n-\mathrm{C}_{18}$ (Cuadro I) asociado con la mayor sensibilidad a la biodegradación de los $n$-alcanos respecto a pristano y fitano (Peters y Moldowan 1993). En el caso de la relación pristano/fitano $(\mathrm{P} / \mathrm{F})$, ésta es similar para el crudo original y luego de los ensayos a 90 días, lo que sugiere que no ocurrió alteración detectable a través de CG-EM de estos isoprenoides acíclicos.

Para los esteranos se observa, después de la biorremediación (Fig. 3b), disminución en la intensidad de las señales para los esteranos de $\mathrm{C}_{21}, \mathrm{C}_{22}$ y $\mathrm{C}_{27}$ a $\mathrm{C}_{29}$, con incremento de la línea base. Sin embargo, el patrón de distribución en cuanto a la intensidad relativa indica que tanto para el crudo original como luego de la biorremediación, los esteranos regulares presentan la siguiente abundancia relativa; $\mathrm{C}_{27}>$ $\mathrm{C}_{28}>\mathrm{C}_{29}$, lo que indica que no ocurrió una perdida preferencial del esterano $\mathrm{C}_{27}$ relativo a $\mathrm{C}_{28}$ y $\mathrm{C}_{29}$, como se ha documentado en crudos biodegradados $\mathrm{y}$ ensayos de biorremediación en productos de refinería (Moldowan et al. 1995).

De acuerdo con Peters et al. (2005), el pregnano $\left(\mathrm{C}_{21}\right)$ y el homopregnano $\left(\mathrm{C}_{22}\right)$ son esteranos regulares más resistentes a la biodegradación que los correspondientes de 27, 28 y 29 átomos de carbono $\left(\mathrm{C}_{27}, \mathrm{C}_{28}\right.$ y $\left.\mathrm{C}_{29}\right)$. Con base en ello, se calculó la relación $\left(\mathrm{C}_{20}+\mathrm{C}_{21}\right) /\left(\mathrm{C}_{27}+\mathrm{C}_{28}+\mathrm{C}_{29}\right)\left[\mathrm{S}\left(\mathrm{C}_{20}-\mathrm{C}_{21}\right)-\right.$

CUADRO I. RELACIONES DE BIOMARCADORES UTILIZADAS PARA INDICAR BIORREMEDIACIÓN.

\begin{tabular}{|c|c|c|c|}
\hline Tipo de biomarcador & Crudo & $\begin{array}{c}\text { Ayacucho } \\
\text { Crudo original* }\end{array}$ & $\begin{array}{c}\text { Ayacucho } \\
\text { Biorremediación t90* }\end{array}$ \\
\hline$n$-alcanos & $\mathrm{C}_{17}-\mathrm{C}_{25} / \mathrm{C}_{26}-\mathrm{C}_{34}{ }^{\mathrm{a}}$ & $2.5 \pm 0.2$ & $1.0 \pm 0.1$ \\
\hline Isoprenoides acíclicos & $\mathrm{P} / \mathrm{F}^{\mathrm{b}}$ & $1.8 \pm 0.2$ & $1.50 \pm 0.04$ \\
\hline$n$-alcanos Isoprenoides acíclicos & $\mathrm{P} / n-\mathrm{C}_{17}{ }^{\mathrm{c}}$ & $2.3 \pm 0.3$ & $2.7 \pm 0.8$ \\
\hline$n$-alcanos Isoprenoides acíclicos & $\mathrm{F} / n-\mathrm{C}_{18}{ }^{\mathrm{c}}$ & $0.77 \pm 0.02$ & $1.2 \pm 0.1$ \\
\hline Esteranos & $\left(\mathrm{C}_{20}+\mathrm{C}_{21}\right) /\left(\mathrm{C}_{27}+\mathrm{C}_{28}+\mathrm{C}_{29}\right)^{\mathrm{e}}$ & $0.05 \pm 0.01$ & $0.3 \pm 0.1$ \\
\hline Terpanos & $\begin{array}{c}\mathrm{C}_{23} \mathrm{TT} / \mathrm{C}_{30} \text {-hopano }^{\mathrm{d}} \\
\% \mathrm{C}_{31}{ }^{\mathrm{f}} \\
\% \mathrm{C}_{32}{ }^{\mathrm{f}} \\
\% \mathrm{C}_{33}{ }^{\mathrm{f}} \\
\% \mathrm{C}_{34}{ }^{\mathrm{f}} \\
\% \mathrm{C}_{35}{ }^{\mathrm{f}} \\
\mathrm{C}_{31} 22 \mathrm{~S} /\left(22 \mathrm{~S}+22 \mathrm{R}{ }^{\mathrm{g}}\right. \\
\mathrm{C}_{32} 22 \mathrm{~S} /(22 \mathrm{~S}+22 \mathrm{R})^{\mathrm{g}} \\
\mathrm{C}_{33} 22 \mathrm{~S} /(22 \mathrm{~S}+22 \mathrm{R})^{\mathrm{g}} \\
\mathrm{C}_{34} 22 \mathrm{~S} /(22 \mathrm{~S}+22 \mathrm{R})^{\mathrm{g}} \\
\mathrm{C}_{35} 22 \mathrm{~S} /(22 \mathrm{~S}+22 \mathrm{R})^{\mathrm{g}}\end{array}$ & $\begin{array}{c}0.87 \pm 0.03 \\
42.3 \\
25.7 \\
16.0 \\
8.7 \\
7.3 \\
0.61 \\
0.64 \\
0.66 \\
0.66 \\
0.67\end{array}$ & $\begin{array}{c}0.5 \pm 0.1 \\
40.9 \\
11.6 \\
17.0 \\
19.6 \\
13.9 \\
0.57 \\
0.57 \\
0.22 \\
0.63 \\
0.60\end{array}$ \\
\hline
\end{tabular}

\footnotetext{
* Los valores de \pm corresponden al error de la medida expresado como desviación del promedio de tres medidas. ${ }^{a} \sum\left(n-\mathrm{C}_{17}\right.$ a $\left.n-\mathrm{C}_{25}\right) / \sum\left(n-\mathrm{C}_{26}\right.$ a $\left.n-\mathrm{C}_{34}\right)$ para los $n$-alcanos en el fragmentograma de masas $m / z=113$. ${ }^{\mathrm{b}} \mathrm{P} / \mathrm{F}=$ pristano/fitano (Didyk et al. 1978).

${ }^{c} \mathrm{P} / n-\mathrm{C}_{17}$ : pristano $/ n-\mathrm{C}_{17}{ }^{\mathrm{c}} \mathrm{F} / n-\mathrm{C}_{18}$ : fitano $/ n-\mathrm{C}_{18}$ (Connan y Cassau 1980 , Peters et al. 1999).

${ }^{\mathrm{d}} \mathrm{C}_{23} \mathrm{TT} / 17 \mathrm{a}$-hopano $=\mathrm{C}_{23}$-terpano tricíclico $/ \mathrm{C}_{30}$-hopano (Peters et al. 2005).

${ }^{\mathrm{e}}\left(\mathrm{C}_{20}+\mathrm{C}_{21}\right) /\left(\mathrm{C}_{27}+\mathrm{C}_{28}+\mathrm{C}_{29}\right)=\mathrm{S}\left(\mathrm{C}_{20}-\mathrm{C}_{21}\right)$-pregnanos/S $\left(\mathrm{C}_{27}-\mathrm{C}_{29}\right)$-esteranos (Zhang et al. 2014).

${ }^{\mathrm{f}}$ Distribución de homohopanos $\% \mathrm{C}_{3 \mathrm{x}}$ (x de 1 a 5) $\left[\mathrm{C}_{3 \mathrm{x}} / \sum \mathrm{C}_{31}-\mathrm{C}_{35}\right] \mathrm{x} 100$ (Peters et al. 2005). Relaciones utilizadas para la figura 6.

${ }^{g} C_{3 x}$ (x de 1 a 5) $=22 S /(22 S+22 R)$ : isomerización de homohopanos (Seifert y Moldowan 1980). Relaciones utilizadas para la figura 7 .
} 
pregnanos/S( $\left.\mathrm{C}_{27}-\mathrm{C}_{29}\right)$-esteranos], la cual incrementa con el avance de la biodegradación (Zhang et al. 2014). Como se puede observar en el cuadro I, los valores de $\left(\mathrm{C}_{20}+\mathrm{C}_{21}\right) /\left(\mathrm{C}_{27}+\mathrm{C}_{28}+\mathrm{C}_{29}\right)$ son $0.05 \pm 0.01 \mathrm{y}$ $0.3 \pm 0.1$ para el crudo original y el sometido a 90 días de biodegradación, respectivamente, lo cual indica que probablemente haya ocurrido alteración de los esteranos. Este aspecto se observa por las diferencias en las distribuciones de las señales entre el crudo original y a los 90 días en la figura 3. El incremento de la relación $\left(\mathrm{C}_{20}+\mathrm{C}_{21}\right) /\left(\mathrm{C}_{27}+\mathrm{C}_{28}+\mathrm{C}_{29}\right)$ es un claro indicativo de la biodegradación o transformaciones internas del crudo, que si bien no se reflejan en cambios significativos de disminución del crudo total, forman parte del proceso de biodegradación a nivel molecular (Zhang et al. 2014).

Para los terpanos, a los 90 días se observa nuevamente una disminución importante de las señales de los hopanos extendidos $\left(\mathrm{C}_{31}\right.$ a $\left.\mathrm{C}_{35}\right)$, con levantamiento de línea base en el cromatograma de masas (Fig. 4b) y disminución de la señal de los terpanos tricíclicos de 25 a 30 átomos de carbono $\left(\mathrm{C}_{25}-\mathrm{C}_{30}\right.$; Fig. 4b). De acuerdo con la literatura (Peters y Moldowan 1993, Peters 2000, Alberdi et al. 2001, Peters et al. 2005, Cheng et al. 2016) los terpanos tricíclicos son más resistentes a la biodegradación en comparación con los pentacíclicos. En este ensayo de biorremediación, la relación $\mathrm{C}_{23} / \mathrm{C}_{30}$ (Cuadro I) disminuye, lo que pareciera indicar que ocurrió la biodegradación tanto de los terpanos pentacíclicos como los tricíclicos. Alberdi et al. (2001) indican que la biodegradación estereoselectiva de terpanos tricíclicos en crudos pesados de los campos costaneros de Bolívar, específicamente del campo Lagunillas, permite detectar la serie completa de terpanos tricíclicos desmetilados, formados en el yacimiento por remoción de grupo metilo del C-10, generando 17-nor-terpanos tricíclicos. Por otra parte, la desmetilación de los terpanos ocurre durante la destrucción de los esteranos y la desmetilación de los hopanos, pero de estos tres grupos de compuestos, los terpanos tricíclicos son más resistentes a la biodegradación. Esto podría sugerir que durante la biorremediación pudo ocurrir alteración de los terpanos tricíclicos.

Con base en la comparación de los iones de $\mathrm{m} / \mathrm{z}$ 191 y 177 de los hopanos extendidos $\left(\mathrm{C}_{31}\right.$ a $\left.\mathrm{C}_{35}\right)$ y 25-norhopanos para el crudo original (Figs. 4a y 5a), y luego del ensayo de biorremediación (Figs. 4b y 5b), se observa que disminuye la intensidad de la señal de estos compuestos y se detectan los hopanos desmetilados $\mathrm{C}_{27}-25,28,30$-trisnorhopano (TNH), $\mathrm{C}_{28}-28,30$-dinorhopano (DNH) y $\mathrm{C}_{29}$ $17 \alpha(\mathrm{H}), 21 \beta(\mathrm{H})-25$-norhopano $(\mathrm{NH})$, tanto en el crudo original como a los 90 días de biodegradación. Esto último podría indicar que los hopanos desmetilados observados están presentes en el crudo original, como consecuencia de su biodegradación en el yacimiento, y no se formaron por alteración del crudo durante los ensayos de biorremediación. Es típico que en crudos biodegradados se observe una distribución en un estado intermedio, con presencia de los hopanos extendidos de $\mathrm{C}_{31}$ a $\mathrm{C}_{35}$, junto con la distribución de los 25-norhopanos de $\mathrm{C}_{26}$ a $\mathrm{C}_{34}(\mathrm{Pe}-$ ters et al. 2005). Éste es el caso del crudo Ayacucho, luego del ensayo de biorremediación a 90 días, donde se observan distribuciones de hopanos extendidos y 25-norhopanos con mayor grado de alteración en cuanto a la ausencia de algunas de sus señales.

Al comparar la distribución de los hopanos extendidos para el crudo original y el obtenido luego del ensayo (Fig. 6) es evidente la variación en la distribución, la cual indica disminución en la intensidad relativa de todos los hopanos extendidos. Adicionalmente, el $\mathrm{C}_{32}$ es menos abundante respecto al $\mathrm{C}_{33}$, al igual que el $\mathrm{C}_{35}$ respecto del $\mathrm{C}_{34}$. De acuerdo con Liao et al (2006), en algunos casos no es evidente el orden de biodegradación de los hopanos extendidos; por ejemplo, durante la degradación microbiana de dos crudos de Venezuela enriquecidos en 25-norhopanos. En estos crudos, luego de cinco semanas de ensayos de biodegradación, se detectó

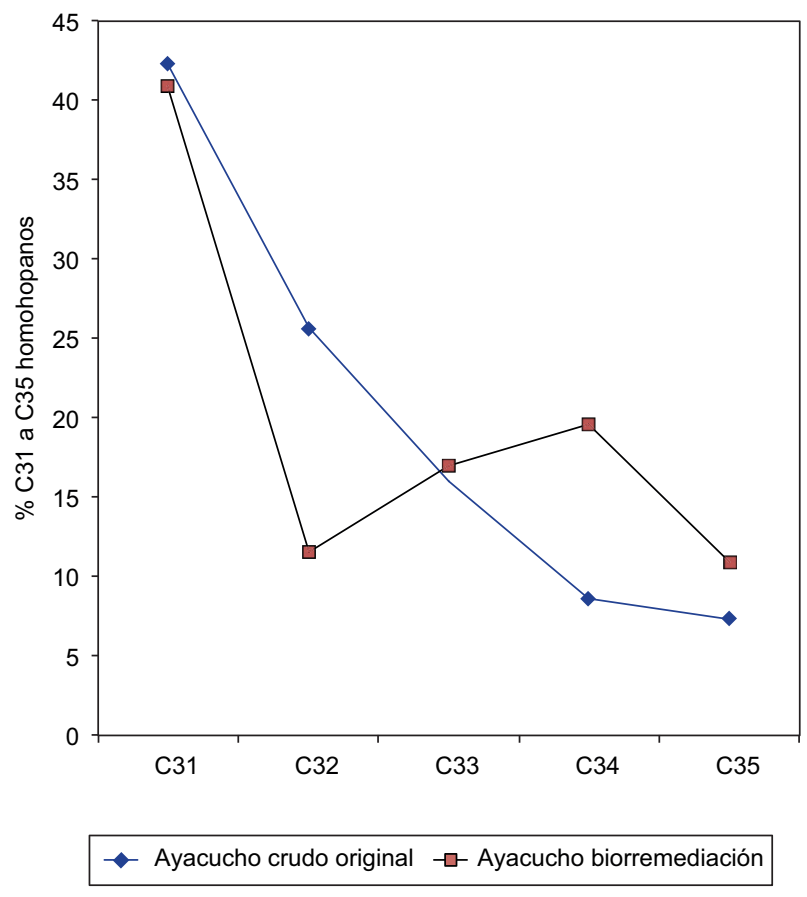

Fig. 6. Distribución de los homohopanos en el crudo Ayacucho original y luego de 90 días de biorremediación. 
que el terpano tricíclico $\mathrm{C}_{28}$ y los hopanos de $\mathrm{C}_{29}-\mathrm{C}_{34}$ $17 \alpha(\mathrm{H}), 21 \beta(\mathrm{H})$ у $\mathrm{C}_{29} 17 \alpha(\mathrm{H}), 21 \beta(\mathrm{H})-25$-norhopano, son alterados, mientras que el $\mathrm{C}_{35} 17 \alpha(\mathrm{H}), 21 \beta(\mathrm{H})$ hopano y el $18 \alpha(\mathrm{H})$-oleanano no lo son. Es posible que, durante los ensayos de biorremediación, el orden de alteración de los hopanos extendidos pueda variar, al igual que la concentración de todos ellos $\left(\mathrm{C}_{31}\right.$ a $\mathrm{C}_{35}$ ) respecto a la observada en el crudo original. $\mathrm{C}_{35}$ es el más alterado, tal como se reporta en la literatura (Peters et al. 2005), pero también puede ocurrir una mayor alteración para el hopano extendido $\mathrm{C}_{32}$. El orden observado a partir del $\mathrm{m} / \mathrm{z} 191$ indica que para los hopanos extendidos la alteración fue: $\mathrm{C}_{35}>\mathrm{C}_{34}<$ $\mathrm{C}_{33}>\mathrm{C}_{32}>\mathrm{C}_{31}$. Este orden es similar al observado en suelos de manglares contaminados con crudo liviano de Arabia (Munoz et al. 1997), y es el orden comúnmente observado en crudos (Peters et al. 2005). Sin embargo, la alteración puede ir por una vía diferente a la desmetilación para formación 25-norhopanos, ya que éstos no se observan en el $\mathrm{m} / \mathrm{z}=177$ del ensayo a los 90 días.

Con el objeto de determinar la posible diferencia de biodegradación entre los isómeros $\mathrm{S}$ y $\mathrm{R}$ de los hopanos extendidos, se realizó el grafico de la figura 7 , donde se representa la relación $22 \mathrm{~S} /(22 \mathrm{~S}+22 \mathrm{R})$ para la serie de $\mathrm{C}_{31}$ a $\mathrm{C}_{35}$ en el crudo Ayacucho original y después del ensayo de biorremediación a



Fig. 7. Distribución de los homohopanos para la relación de isomerización $[22 \mathrm{~S} /(22 \mathrm{~S}+22 \mathrm{R})]$ en el crudo Ayacucho original y luego de 90 días de biorremediación.
90 días. De acuerdo con la literatura sobre crudos y ensayos de laboratorio, el isómero biológico de los hopanos desmetilados $(17 \alpha(\mathrm{H})$-homohopanos $22 \mathrm{R})$ es más sensible a la biodegradación que el isómero químico (17 $\alpha(\mathrm{H})$-homohopanos 22S) (Munoz et al. 1997, Requejo y Halpern 1989, Bost et al. 2001, Peters et al. 2005). Sin embargo, se ha reportado en crudos del campo Cymric (California), con un alto nivel de biodegradación, que el isómero $22 \mathrm{~S}$ es de concentración comparable en los hopanos extendidos $C_{31}, C_{32}, C_{34}$ y $C_{35}$, pero de menor concentración para el $\mathrm{C}_{33}$, lo que produce una disminución en la relación $22 \mathrm{~S} /(22 \mathrm{~S}+22 \mathrm{R})$, para este homohopano (Peters et al. 2005). Este resultado parece sugerir una degradación selectiva del isómero $22 \mathrm{~S}$ del homohopano $\mathrm{C}_{33}$ durante los ensayos de biorremediación; sin embargo, se requiere de nuevos ensayos para corroborar este resultado.

\section{CONCLUSIONES}

De acuerdo con el análisis de los biomarcadores de la fracción de hidrocarburos saturados ( $n$-alcanos, pristano, fitano, terpanos y esteranos), se determinó que el crudo Ayacucho utilizado para los ensayos de biorremediación está biodegradado. La presencia de biomarcadores con distinta sensibilidad a la biodegradación ( $n$-alcanos, pristano, fitano, junto con hopanos desmetilados), sugiere una mezcla de crudos en el yacimiento, producto de distintas cargas de crudos sometidas a biodegradación.

La concentración de COT remanente, luego de la extracción del crudo a $t_{1}$, presenta un leve incremento respecto al suelo sin crudo, debido posiblemente al crudo remanente en el suelo, que no puede extraerse con el cloruro de metileno. Además, también puede ocurrir extracción de lípidos y otros compuestos orgánicos propios de la materia orgánica del suelo (restos vegetales, carbono microbiano). A partir de los 30 días y hasta los 90, permanece constante, indicando que no hay cambios en el COT remanente.

Durante la biorremediación no ocurrió la formación de 25-norhopanos; sin embargo, el orden de biodegradación para los hopanos extendidos fue: $\mathrm{C}_{35}>\mathrm{C}_{34}<\mathrm{C}_{33}>\mathrm{C}_{32}>\mathrm{C}_{31}$. Esto sugiere una mayor alteración para los homohopanos $\mathrm{C}_{35}$ y $\mathrm{C}_{32} \mathrm{y}$ en los isómeros $\mathrm{S}$ y $\mathrm{R}$, una degradación selectiva del isómero 22S del homohopano $\mathrm{C}_{33}$.

Se sugiere alteración de los terpanos tricíclicos durante la biorremediación, relacionada con la variación en la intensidad de la señal del crudo original y luego de la biorremediación. 
Los esteranos no presentan un orden de alteración específico luego de los 90 días de biorremediación; sin embargo, la intensidad de las señales entre $C_{21}$ y $\mathrm{C}_{22}$ respecto a $\mathrm{C}_{27}, \mathrm{C}_{28}$ y $\mathrm{C}_{29}$, sugiere una posible alteración por biodegradación.

A nivel molecular, la transformación debida a la biodegradación para el crudo Ayacucho indica: 1) remoción de los alcanos $<n-\mathrm{C}_{25}$, sin alteración $>n-\mathrm{C}_{25}$ y los isoprenoides pristano y fitano; 2) alteración de los terpanos tricíclicos y los hopanos extendidos, y 3) alteración de los esteranos. Estos cambios o alteraciones son un reflejo de las modificaciones internas que pueden ocurrir a un crudo extrapesado o al desecho de una fosa petrolizada durante un proceso de biorremediación, sin que llegue a obtenerse una disminución significativa del crudo total para cumplir con la meta final de la biorremediación: la reducción a concentraciones aceptadas por la legislación acorde con criterios de limpieza.

\section{AGRADECIMIENTOS}

Al Consejo de Desarrollo Científico y Humanístico de la UCV por el financiamiento otorgado a través del proyecto Estudio de la biodegradación de crudos livianos y pesados en suelos de diferente textura y composición (PI-03-00-7178-2008). Al Dr. Salvador Lo Mónaco por su apoyo en la realización de los análisis de textura y composición mineralógica del suelo.

\section{REFERENCIAS}

Ali N., Dashti N., Khanafer M., Al-Awadhi H. y Radan S. (2020). Bioremediation of soils saturated with spilled crude oil. Sci. Rep. 10, 1116. https://doi.org/10.1038/ s41598-019-57224-x

Alberdi M., López C. y Galarraga F. (1996). Genetic classification of crude oil families in the Eastern Venezuelan basin. Boletín de la Sociedad Venezolana de Geólogos 21, 7-21.

Alberdi M., Moldowan J.M., Peters K.E. y Dahl J.E. (2001). Stereoselective biodegradation of tricyclic terpanes in heavy oils from the Bolívar Coastal Fields, Venezuela. Org. Geochem. 32, 181-191. https://doi. org/10.1016/S0146-6380(00)00130-3

Bost F.D., Frontera-Suau R., McDonald J.T., Peters K.E. y Morris P.J. (2001). Aerobic biodegradation of hopanes and norhopanes in Venezuelan crude oil. Org. Geochem. 37, 105-114. https://doi.org/10.1016/ S0146-6380(00)00147-9
Brown D.M., Matthijs Bonte R.G., James D. y Boogaard P.J. (2017). Heavy hydrocarbon fate and transport in the environment. Q. J. Eng. Geol. Hydroge. 50, 333346. https://doi.org/10.1144/qjegh2016-142

Casas J., González M. y Marfisi N. (2007). Interpretación de facies genéticas en pozos verticales/inclinados/horizontales y su integración en el modelo geológico. Formación Oficina, campo Sincor, Bloque Junín, Faja Petrolífera del Orinoco, Venezuela. IX Congreso Geológico Venezolano. Facultad de Ingeniería, Universidad Central de Venezuela, Caracas, octubre, $12 \mathrm{pp}$.

Cheng X., Hou D., Xu C. y Wang F. (2016). Biodegradation of tricyclic terpanes in crude oils from Bohai Bay Basin. Org. Geochem. 101, 11-21. https://doi. org/10.1016/j.orggeochem.2016.08.007

Connan J. y Cassau A. (1980). Properties of gas petroleum liquids derived from terrestrial kerogen at various maturation levels. Geochim. Cosmochim. Ac. 44, 1-23. https://doi.org/10.1016/0016-7037(80)90173-8

Córdova A., Infante C., López L. y Lugo P. (2013). Efecto de la concentración inicial de crudo en la biorremediación de un suelo contaminado. Geos 44, 115-120.

Didyk B.M., Simoneit B.R.T., Brassell S.C. y Eglinton G. (1978). Organic geochemical indicators of palaeoenvironmental conditions of sedimentation. Nature 272, 216-222. https://doi.org/10.1038/272216a0

Erdogan E. y A. Karaca. (2011). Bioremediation of crude oil polluted soils. Asian Journal of Biotechnology 3 (3) 206-213. https://doi.org/10.3923/ajbkr.2011.206.213

García M.G., Infante C. y López L. (2012). Biorremediación de un crudo mediano en suelos con diferente textura con y sin agente estructurante. BioAgro 24 (2), 93-102.

Gough M.A. y Rowland S.J. (1990). Characterization of unresolved complex mixture of hydrocarbons in petroleum. Nature 334 648-650. https://doi. org $/ 10.1038 / 344648 \mathrm{a} 0$

Hesse P.R. (1971). A textbook of soil chemical analysis. John Murray Publishers, Londres, Reino Unido, 520 pp.

Infante C. (2001). Biorrestauración de áreas impactadas por crudo por medio de INTEBIOS ${ }^{\mathrm{R}}$ y BIORIZE $^{\mathrm{R}}$. Interciencia 26 (10), 503-507.

Infante C. (2005). Contaminación de suelos y recuperación ecológica en Venezuela. Acta Biol. Venez. 25 (1-2), 43-49.

Infante C., Morales F., Ehrmann U., Hernández-Valencia I. y León N. (2010a). Hydrocarbon bioremediation and phytoremediation in tropical soils: Venezuelan study case. En: Trends in bioremediation and phytoremediation (Płaza G., Ed.). Research Signpost, Thiruananthapuram, India, 429-451. 
Infante C., Ortega C., Morales F., Ehrmann U., HernándezValencia I. y Pérez R. (2010b). Efecto del potasio sobre el proceso de biorremediación de un suelo contaminado con un crudo mediano. BioAgro 22 (2), 145-152.

Killops S.D. y al-Juboori M.A. (1990). Characterisation of the unresolved complex mixture (UCM) in the gas chromatograms of biodegraded petroleums. Org. Geochem. 15, 147-160. https://doi.org/10.1016/01466380(90)90079-F

Larter S., Wilhelms I., Head I., Koopman M., Aplin A., Di Primio R., Zwach C., Erdmann M. y Telnaes N. (2003). The controls on the composition of biodegraded oils in the deep subsurface: Part I-Biodegradation rates in petroleum reservoirs. Org. Geochem. 34 (4), 601-613. https://doi.org/10.1016/S0146-6380(02)00240-1

Larter S., Huang H., Adams J., Bennett B., Jokanola O., Oldenburg T., Jones M., Head I., Riediger C. y Fowler M. (2006). The controls on the composition of biodegraded oil in the deep surface: Part II-Geological controls on subsurface biodegradation fluxes and constraints on reservoir-fluid property prediction. Am. Assoc. Petr. Geol. B. 90, 921-938. http://dx.doi. org $/ 10.1306 / 01270605130$

Liao Z., Geng A., Graciaa A., Creux P., Chrostowska A. y Zhang Y. (2006). Saturated hydrocarbons occluded inside asphaltene structures and their geochemical significance, as exemplified by two Venezuelan oils. Org. Geochem. 37, 291-303. https://doi.org/10.1016/j. orggeochem.2005.10.010

López L. y Lo Mónaco S. (2010). Geoquímica de crudos de la Faja Petrolífera del Orinoco. Cuenca Oriental de Venezuela. Revista de la Facultad de Ingeniería 25, 41-50.

López L. 2013. Biomarcadores. Aplicaciones en la geoquímica del petróleo. Ediciones de la Biblioteca, Universidad Central de Venezuela, Caracas, 46 pp.

López L. (2014). Study of the biodegradation levels of oils from the Orinoco Oil Belt (Junín area) using different biodegradation scales. Org. Geochem. 66, 60-69. https://doi.org/10.1016/j.orggeochem.2013.10.014

López L., Lo Mónaco S. y Volkman J. (2015). Evidence for mixed and biodegraded crude oils in the Socororo field, Eastern Venezuela Basin. Org. Geochem. 82, 1221. https://doi.org/10.1016/j.orggeochem.2015.02.006

López L. y Lo Mónaco S. (2017). Vanadium, nickel and sulfur in crude oils and source rocks and their relationship with biomarkers: Implications for the origin of crude oils in Venezuelan basins. Org. Geochem. 104, 53-68. https://doi.org/10.1016/j.orggeochem.2016.11.007

Moldowan J.M., Dahl J., McCaffrey M.A., Smith W.J. y Fetzer J. (1995). Application of biological markers technology to biorremediation of refinery by-products.
Energ. Fuel. 9, 155-162. https://doi.org/10.1021/ ef00049a023

Munoz D., Guiliano M., Doumenq P., Jacquod F., Scherrer P. y Mille G. (1997). Long term evolution of petroleum biomarkers in mangrove soil (Guadalupe). Mar. Pollut. Bull. 34, 868-874. https://doi.org/10.1016/S0025$326 X(97) 00061-1$

Pardo E., Casas J., Marcos J., Delgado D., Expósito M. y Bellorini J.P. (2007). Integración de la data fisicoquímica y geológica para la construcción del modelo del acuífero de SINCOR, Faja del Orinoco, Venezuela. IX Congreso Geológico Venezolano. Facultad de Ingeniería, Universidad Central de Venezuela, Caracas, octubre, $12 \mathrm{pp}$.

Peters K.E. y Moldowan J.M. (1993). The biomarker guide. Interpreting molecular fossils in petroleum and ancient sediments. Prentice Hall, New Jersey, 363 pp.

Peters K.E., Frase H., Amis W., Rustanto B. y Hermento E. (1999). Geochemistry of crude oils from Eastern Indonesia. Am. Assoc. Petr. Geol. B. 83(12), 19271942. https://doi.org/10.1306/E4FD4643-1732-11D7$8645000102 \mathrm{C} 1865 \mathrm{D}$

Peters K.E. (2000). Petroleum tricyclic terpanes: Predicted physicochemical behavior from molecular mechanics calculations. Org. Geochem. 31, 497-507. https://doi. org/10.1016/S0146-6380(00)00029-2

Peters K.E., Walters C. y Moldowan J. (2005). The biomarker guide. Cambridge. University Press Cambridge $706 \mathrm{p}$.

Requejo A.G. y Halpen H.I. (1989). An unusual hopane biodegradation sequence in tar sands from the Pt arena (Monterrey) Formation. Nature 342, 670-673. https:// doi.org/10.1038/342670a0

Seifert W.K. y Moldowan J.M. (1980). The effect of thermal stress on source rock quality as measured by hopane stereochemistry. En: Advances in organic geochemistry 1979 (Douglas A.G. y Maxwell J.R., Eds.). Pergamon Press, Oxford, 229-237. https://doi. org/10.1016/0079-1946(79)90107-1

Sutton P.A., Lewis C.A. y Rowland S.J. (2005). Isolation of individual hydrocarbons from the unresolved complex hydrocarbon mixture of a biodegraded crude oil using preparative capillary gas chromatography. Org. Geochem. 36, 963-970. https://doi.org/10.1016/j.orggeochem.2004.11.007

Tissot B.P. y Welte, D.H. (1984) Petroleum formation and occurrence. 2a ed. Springer-Verlag, Berlín, 699 pp.

Volkman J.K., Alexander R., Kagi R.I., Noble R.A. y Woodhouse G.W. (1983a). A geochemical reconstruction of oil generation in the Barrow Sub-basin of Western Australia. Geochim. Cosmochim. Ac. 47 (12), 2091-2105. https://doi.org/10.1016/00167037(83)90034-0 
Volkman J.K., Alexander R., Kagi R.I. y Woodhouse C.W. (1983b.) Demethylated hopanes in crude oils and their applications in petroleum geochemistry. Geochim. Cosmochim. Ac. 47 (4), 785-794. https:// doi.org/10.1016/0016-7037(83)90112-6

Volkman J.K., Alexander R., Kagi R.I., Rowland S.J. y Sheppard P.N. (1984). Biodegradation of aromatic hydrocarbons in crude oils from the Barrow Sub-basin of Western Australia. Org. Geochem. 6, 619-632. https:// doi.org/10.1016/0146-6380(84)90084-6

Wenger L.M., Davis C.L. e Isaksen G.H. (2002). Multiple controls on petroleum biodegradation and impact on oil quality. Spe. Reserv. Eval. Eng. 5, 375-383. https:// doi.org/10.2118/80168-PA
Wong-Ng W., McMurdie H.F., Hubbard C.R. y Mighell A.D. (2001). JCPDS-ICDD Research Associateship (cooperative program with NBS/NIST). J. Res. Natl. Inst. Stan. 106 (6), 1013-1028. https://doi.org/10.6028/ jres. 106.052

Zhang S., Huang H., Su J., Zhu G., Wang X. y Larter S. (2014). Geochemistry of paleozoic marine oils from the Tarim Basin, NW China. Part 4: Paleobiodegradation and oil charge mixing. Org. Geochem. 67, 41-57. https://doi.org/10.1016/j.orggeochem.2013.12.008 